\title{
Long-term effects of preterm birth on behavior and neurosteroid sensitivity in the guinea pig
}

\author{
Julia C. Shaw ${ }^{1,2}$, Hannah K. Palliser ${ }^{1,2}$, Rebecca M. Dyson ${ }^{3}$, Jonathan J. Hirst ${ }^{1,2}$ and Mary J. Berry ${ }^{4,5}$
}

BACKGROUND: Ex-preterm children and adolescents are at risk of developing late-onset neurodevelopmental and behavioral disorders. The mechanisms by which this happens are poorly understood and relevant animal models are required.

METHODS: Ex-preterm (delivered at $62 \mathrm{~d}$ gestation) and term (spontaneously delivered) juvenile guinea pigs underwent behavioral testing at $25 \mathrm{~d}$ corrected postnatal age, with tissues collected at $28 \mathrm{~d}$. Neurodevelopmental markers (myelin basic protein (MBP) and glial fibrillary acidic protein (GFAP)) were analyzed in the hippocampus and subcortical white matter by immunohistochemistry. Gamma-aminobutyric acid A $\left(G A B A_{A}\right)$ receptor subunit mRNA levels were quantified by reverse transcription polymerase chain reaction (RT-PCR), and salivary cortisol measured by enzyme-linked immunosorbent assay.

RESULTS: Preterm males travelled greater distances, were mobile for longer, spent more time investigating objects, and approached or interacted with familiar animals more than controls. Myelination and reactive astrocyte coverage was lower in the hippocampus and the subcortical white matter in preterm males. Hippocampal levels of the $\alpha 5$ subunit were also lower in the preterm male brain. Baseline salivary cortisol was higher for preterm males compared to controls.

CONCLUSION: We conclude that juvenile ex-preterm male guinea pigs exhibit a hyperactive phenotype and feature impaired neurodevelopment, making this a suitable model for future therapeutic studies.

C hildren born preterm (birth at $<37 \mathrm{wk}$ gestation) have an increased risk of developing a long-term neurodevelopmental disability $(1,2)$. Importantly, this risk exists even in those thought to be "well" at the time of discharge from neonatal care and in those with no evidence of the structural brain injuries known to be associated with adverse neurodevelopmental outcomes (e.g., intraventricular hemorrhage or periventricular leukomalacia) $(3,4)$. Thus, although intraventricular hemorrhage and, or, periventricular leukomalacia explain neurodevelopmental problems in a subset of high-risk infants, they do not account for the overall burden of neurodisability seen in the ex-preterm population.

Although major neurodevelopmental problems are usually picked up early, subtle behavioral or psychiatric disorders may not become apparent until school age, at a time distant from the causative insult $(5,6)$. Anxiety disorder and attention deficit hyperactivity disorder are the most commonly diagnosed disorders in school-aged ex-preterm children $(7,8)$. Attention deficit hyperactivity disorder has a male preponderance and is characterized by a deficit in behavioral inhibition, inattention, impulsivity and social difficulties, whereas anxiety disorder is more commonly diagnosed in ex-preterm females $(7,8)$. Thus, the behavioral outcomes of preterm birth occur in a sex-dependent manner. Other neuropathologies, including depression, and impaired cognitive performance are also increased in those born preterm compared to children and adolescents born at term (5,9-11). Hypo-myelination of white matter tracts with reduction in the white matter volume of brain regions such as the hippocampus and frontal cortex is also described in expreterm neonates, children, and adolescents (12-14). Despite the clear link between preterm birth and later neurological impairment, the causal mechanisms underpinning pretermassociated brain injury remain poorly understood.

One possible mechanism linking preterm birth with the development of these neurological morbidities and accompanying white matter reductions may be the early loss of exposure to neurosteroids. Neurosteroids, particularly allopregnanolone, are present in high levels during fetal life, especially the latter stages of gestation. Preterm birth leads to a premature loss of placental support of allopregnanolone synthesis and a decline in plasma and brain levels in the newborn compared to the fetus at equivalent postconceptual age (15). Allopregnanolone acts as an agonist at the extrasynaptic $\mathrm{GABA}_{\mathrm{A}}$ receptor and exerts an inhibitory effect, suppressing excessive neuronal excitation and maintaining tonic enhancement of GABA-mediated synaptic inhibition (15-17).

The $\mathrm{GABA}_{\mathrm{A}}$ receptor composition has a key role in regulating neurosteroid action. Receptors containing the $\delta$ and $\alpha 4-6$ subunits are highly sensitive to allopregnanolone concentrations

\footnotetext{
${ }^{1}$ School of Biomedical Sciences and Pharmacy, University of Newcastle, Newcastle, Australia; ${ }^{2}$ Hunter Medical Research Institute, Mothers and Babies Research Centre, Newcastle, Australia; ${ }^{3}$ Department of Paediatrics, Graduate School of Medicine and IHMRI, University of Wollongong, Wollongong, Australia; ${ }^{4}$ Centre for Translational Physiology, University of Otago, Wellington, New Zealand; ${ }^{5}$ Department of Paediatrics and Child Health, University of Otago, Wellington, New Zealand. Correspondence: Julia C. Shaw (Julia.shaw@uon.edu.au)

Received 14 October 2015; accepted 28 January 2016; advance online publication 4 May 2016. doi:10.1038/pr.2016.63
} 


\section{Articles | Shaw etal.}

observed in the fetal brain and this interaction has been suggested to be integral to proper neurodevelopment $(16,18)$. The receptors are highly expressed throughout the brain, including on myelinating oligodendrocytes, however regional variation exists with subunits such as the $\delta$ and $\alpha 4-5$ subunits highly expressed in the hippocampus specifically $(15,17)$. Mouse knockout studies involving these subunits result in increased anxietylike behavior and seizure susceptibility $(19,20)$. Interestingly, in humans markedly lower levels of the $\delta$ subunit are observed in the frontal cortex in patients with major depressive disorder $(21,22)$. Additionally, lower levels of both the $\alpha 5$ and $\delta$ subunits are present following chronic stress and in patients suffering post-traumatic stress disorder and anxiety, implicating decreased expression of these subunits with major depressive disorders $(23,24)$. Previously, we have reported that the expression of these subunits differed between the preterm and term brain at the time of delivery, or allopregnanolone withdrawal, in guinea pigs. Allopregnanolone withdrawal resulted in an adaptive increase in the expression of the $\delta$ and $\alpha 6$ subunits in the brain of term guinea pig neonates, and thus represents the feedback impact of allopregnanolone levels on $\mathrm{GABA}_{\mathrm{A}}$ receptor subunit expression (25). This increase was seemingly absent in the preterm cohort, with expression remaining unchanged between the preterm fetal and neonatal animals $24 \mathrm{~h}$ after birth. Currently, it is unknown whether these changes in expression persist into childhood, or are rectified in the initial neonatal period.

Our previous studies have demonstrated a dramatic drop in brain allopregnanolone concentrations following birth at term and preterm compared to fetal levels (26). This reduced allopregnanolone-mediated neuroprotection increases the vulnerability of the neonatal brain to insults such as hypoxia. Furthermore, these preterm animals also show decreased myelination in the CA1 region of the hippocampus and the adjacent subcortical white matter $24 \mathrm{~h}$ after delivery, and in the cerebellum at term equivalence (25-27).

In this study, we examined the effect of preterm birth on brain development, focusing on the mature myelinating oligodendrocytes and reactive astrocytes that are essential for proper neuronal circuitry and synaptic transmission, to identify effects at adolescence in guinea pigs. We also aimed to ascertain the impact that preterm delivery has on the expression of neurosteroid-sensitive $\mathrm{GABA}_{\mathrm{A}}$ receptor subunits, in addition to behavior at juvenility, with a particular focus on the hyperactive and anxious behaviors that are common to ex-preterm males and females respectively. We hypothesize that early loss of neurosteroid supply alters $\mathrm{GABA}_{\mathrm{A}}$ receptor subunit expression, which then contributes to immature and delayed neurodevelopment following preterm delivery. We further hypothesize that these changes in neurosteroid sensitivity and neurodevelopment, specifically reduced oligodendrocyte and astrocyte expression, will contribute to the development of a hyperactive phenotype and an anxious phenotype, in male and female ex-preterm juveniles respectively, in a sex-dependent manner.

\section{RESULTS}

\section{Physical Characteristics}

The average litter size across the term and preterm deliveries was three, and the number of pups that were either stillborn or were euthanized in the early neonatal period was approximately $10 \%$ for term pups and 30\% for preterm pups.

Mean gestational age at delivery and birth, postmortem, and organ weights for juveniles collected at corrected postnatal day 28 are depicted in Table 1 . Within each sex, birth weights of the preterm neonates were lower (males $P=0.0135$, females $P=0.002$ ). No difference in postmortem weight was observed, however the interaction between gestational age and sex was significant $(P=0.0213)$. Percentage of weight gain from birth until postmortem was higher for the preterm males and females compared to their term counterparts (males $P<0.0001$, females $P=0.0013$ ). The preterm males also had a higher percentage of weight gain than the preterm females $(P=0.0027)$. Liver weight was significantly higher for the preterm males compared to the term males $(P=0.035)$, and was also higher than the preterm females $(P=0.0029)$. The interaction between gestational age and sex for liver weight was also significant $(P=0.0151)$.

\section{Behavior}

Open field and environment exploration. Distance travelled in the open-field arena (Figure 1a) and inner zone (Figure 1b) was higher for preterm males at corrected postnatal day 25 compared to term males $(P=0.026$ and $P=0.046$ respectively). The time mobile in both the open-field arena (Figure 1c) and inner zone (Figure 1d) was higher for preterm males $(P=0.036$ and $P=0.021$ respectively). Term females exhibited higher distances travelled in both the open field and inner zone, and more time spent mobile in the open field compared to term males $(P=0.0065, P=0.026$, and $P=0.049$ respectively). Preterm males spent more time

Table 1. Juvenile physical characteristics

\begin{tabular}{|c|c|c|c|c|c|c|c|c|c|}
\hline & Pups (n) & Dams $(n)$ & $\begin{array}{c}\text { Gestational } \\
\text { age delivery }\end{array}$ & $\begin{array}{c}\text { Birth } \\
\text { weight (g) }\end{array}$ & $\begin{array}{l}\text { Postmortem } \\
\text { weight (g) }\end{array}$ & $\begin{array}{c}\text { Brain } \\
\text { weight (g) }\end{array}$ & $\begin{array}{l}\text { Hippocampus } \\
\text { weight }(\mathrm{g})\end{array}$ & $\begin{array}{l}\text { Half cerebellum } \\
\text { weight (g) }\end{array}$ & $\begin{array}{c}\text { Liver } \\
\text { weight (g) }\end{array}$ \\
\hline Term male & 10 & 8 & $68.87 \pm 0.29$ & $94.2 \pm 4.22$ & $270.1 \pm 10.79$ & $3.36 \pm 0.042$ & $0.12 \pm 0.0041$ & $0.53 \pm 0.055$ & $12.12 \pm 0.57$ \\
\hline Preterm male & 10 & 8 & $62 \pm 0^{+}$ & $76.35 \pm 1.88^{\dagger}$ & $322 \pm 7.28$ & $3.32 \pm 0.047$ & $0.12 \pm 0.0075$ & $0.45 \pm 0.031$ & $15.26 \pm 0.79^{t, t}$ \\
\hline Preterm female & 8 & 8 & $62 \pm 0^{+}$ & $74.51 \pm 5.16^{+}$ & $265.75 \pm 21.7$ & $3.18 \pm 0.082$ & $0.12 \pm 0.0048$ & $0.42 \pm 0.026$ & $10.88 \pm 0.66$ \\
\hline
\end{tabular}

Values are expressed as mean \pm SEM and are calculated for animal numbers.

${ }^{\dagger}$ Denotes a significant difference between preterm and term within a sex, whereas ${ }^{\ddagger}$ denotes a significant difference between sexes within a gestational age group $(P<0.05)$. 
investigating the foreign objects placed into the open-field arena compared to term males (Figure 1e, $P=0.036$ ).

Social interaction test. The number (Figure 2a), and frequency of approaches (Figure 2b), toward a familiar animal in the arena was higher for preterm males compared to term counterparts $(P=0.0072$, and $P=0.0062$ respectively). Similarly, the number (Figure 2c) and frequency (Figure 2d) of affectionate interactions toward the familiar animal was also higher for preterm males compared to terms $(P=0.033$, and $P=0.034$ respectively). There were no differences identified for number or frequency of agonistic interactions (data not shown).

\section{Immunohistochemistry}

Oligodendrocytes. Mature oligodendrocyte cell areas were compared by immunostaining for MBP. MBP area coverage

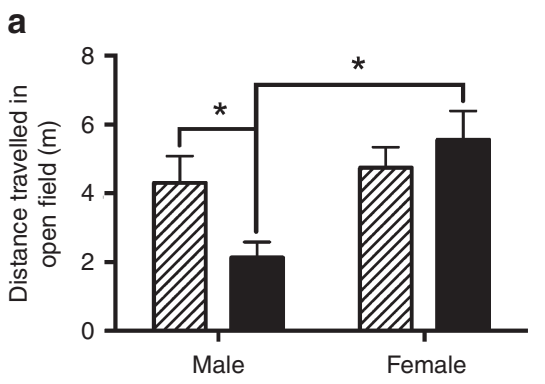

d b

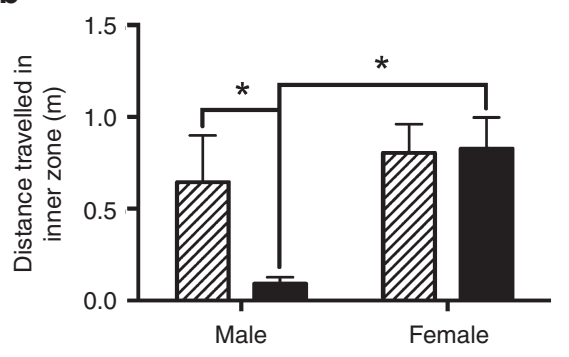

C

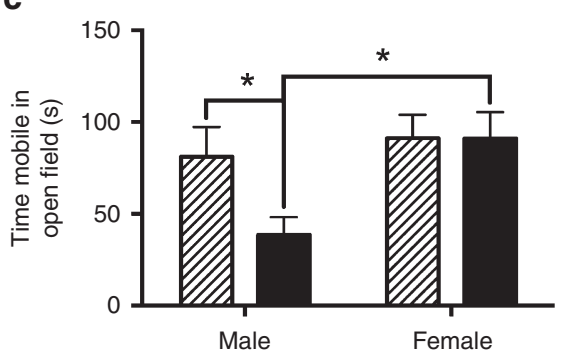

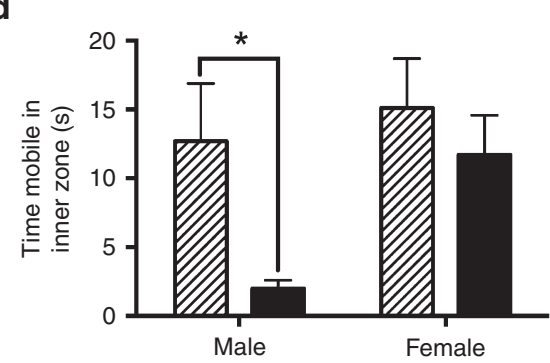

e

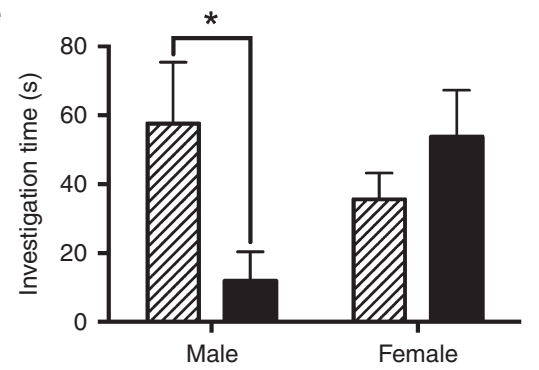

Figure 1. Open field and environment exploration behavioral measurements. (a) Distances travelled by preterm and term juvenile guinea pigs at corrected postnatal day 25 in the open-field arena and (b) the inner zone. (c) Time spent mobile in the open-field arena and (d) the inner zone. (e) Total time spent investigating foreign objects in the open-field arena. Mean \pm SEM. Preterm $=$ hashed bars, term $=$ black bars; ${ }^{*}$ indicates $P<0.05$ between bars linked by black lines. Animal numbers are preterm males $=10$, term males $=10$, preterm females $=8$, and term females $=10$ for $\mathbf{a}$ - $\mathbf{d}$. Animal numbers are preterm males $=8$, term males $=6$, preterm females $=6$, and term females $=10$ for $\mathbf{e}$ ).

a

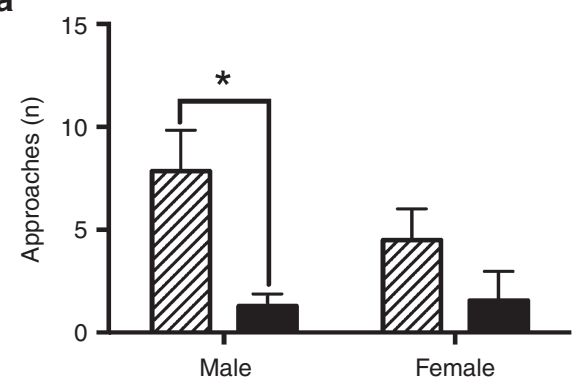

C

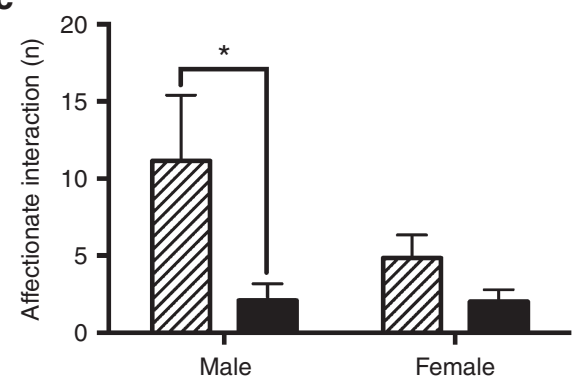

b

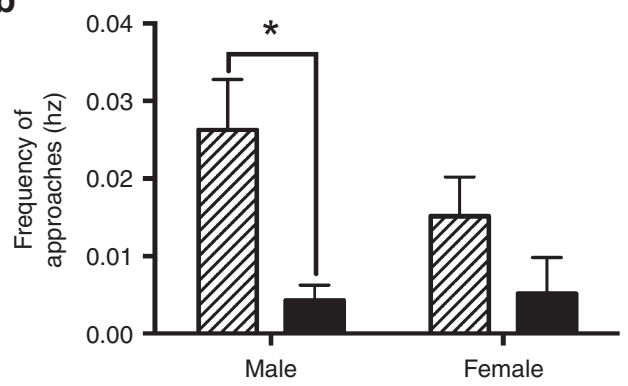

d

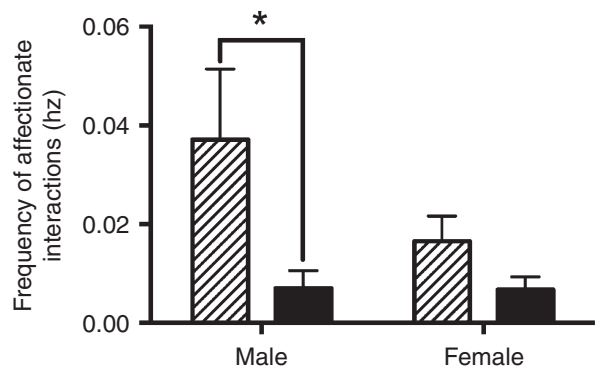

Figure 2. Social interaction behavioral measurements. (a) Total number and (b) frequency of approaches, and affectionate interactions (c and $\mathbf{d}$ respectively) toward a familiar animal by preterm and term juvenile guinea pigs at corrected postnatal day 25 . Mean \pm SEM. Preterm $=$ hashed bars, term $=$ black bars; * indicates $P<0.05$ between bars linked by black lines. Animal numbers are preterm males $=7$, term males $=10$, preterm females $=6$, and term females $=7$. 


\section{Articles | Shaw etal.}

a

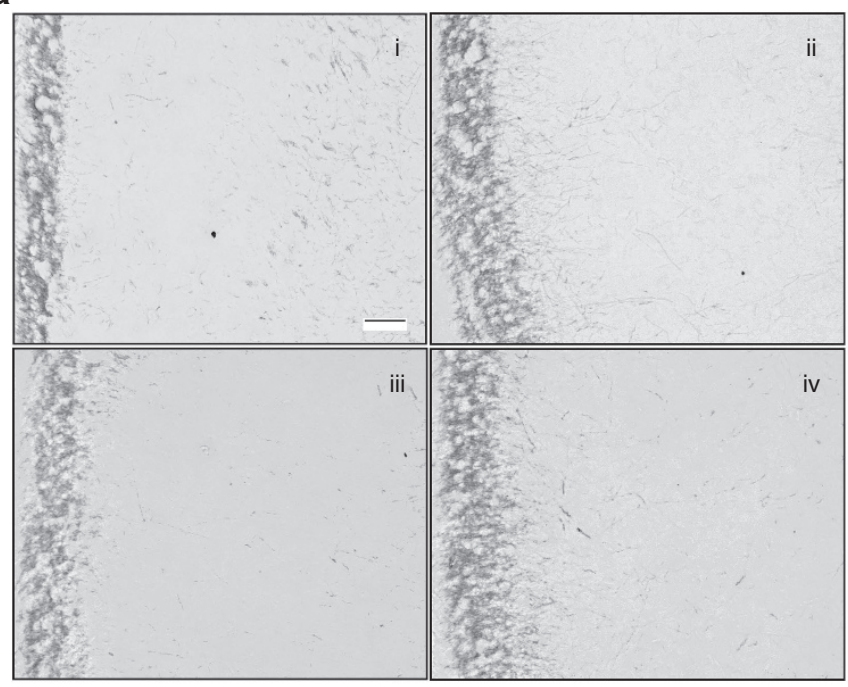

c

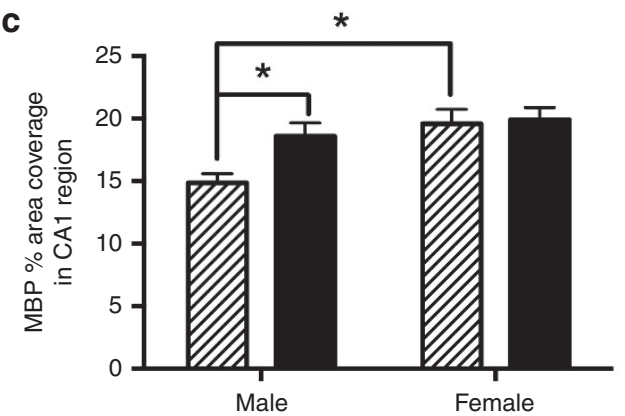

b

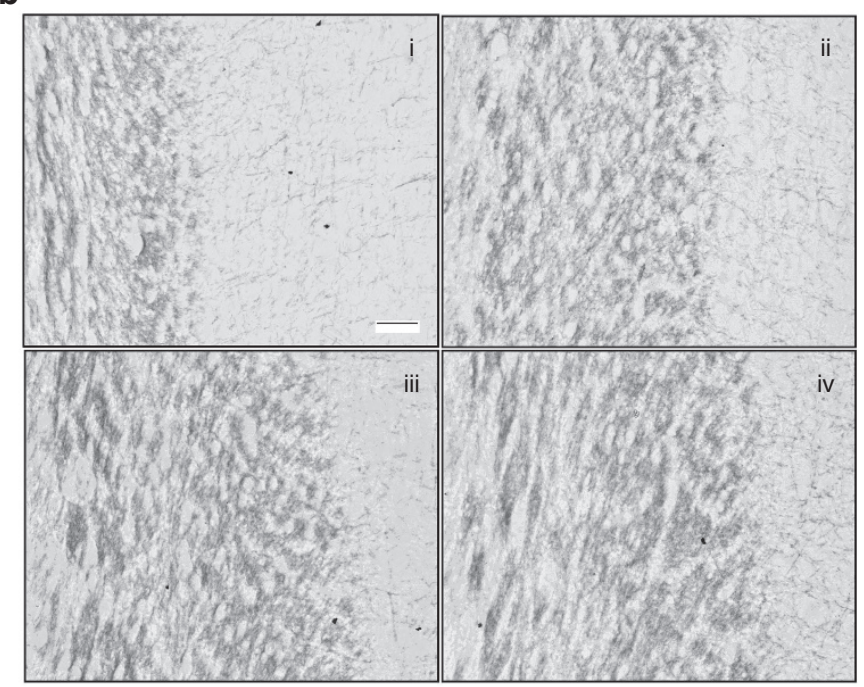

d

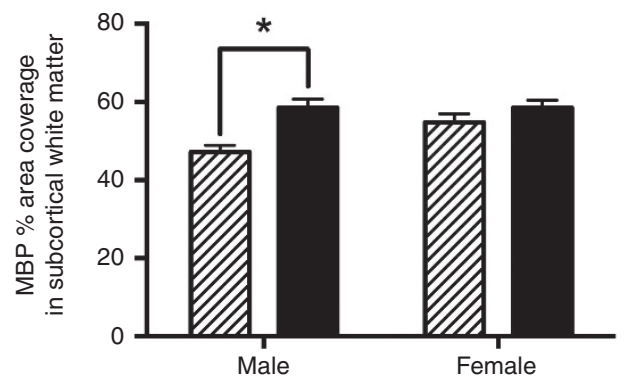

Figure 3. Myelin basic protein immunolabeling of juvenile guinea pig brain regions. Representative photomicrographs at $20 \times$ magnification of myelin basic protein immunolabeling and percent coverage in the juvenile guinea pig external capsule adjacent to the CA1 region of the dorsal hippocampus (a and $\mathbf{c}$ ) and the adjacent subcortical white matter (b and d). Scale bar = $50 \mu$ m; (i) male preterm, (ii) male term, (iii) female preterm, and (iv) female term for all photomicrographs. Mean \pm SEM. Preterm = hashed bars, term = black bars; * indicates $P<0.05$ between bars linked by black lines. Animal groups are preterm males $=8$, term males $=6$, preterm females $=6$, and term females $=7$.

in the CA1 region of the dorsal hippocampus was lower for preterm males compared to both their term counterparts (Figure 3c, $P=0.05)$, and to preterm females $(P=0.0098)$. In the immediately adjacent subcortical white matter, preterm males exhibited decreased area coverage of MBP compared to term males (Figure 3d, $P=0.0019$ ).

Astrocytes. The areas occupied by reactive astrocytes were compared by immunostaining for GFAP. GFAP area coverage in the CA1 region of the dorsal hippocampus was lower in the preterm males compared to both their term counterparts (Figure $4 c, P=0.032)$, and to preterm females $(P=0.012)$. Interaction between gestational age and sex was also significant in the CA1 region $(P=0.010)$. In the adjacent dentate gyrus, males exhibited decreased area coverage of GFAP compared to the females (Figure 4d, $P=0.023$ ). Subcortical white matter expression was also analyzed, however, there were no significant differences identified between sexes or gestational ages (data not shown).

\section{GABA $_{A}$ Receptor Subunit Expression}

In hippocampal tissues, males had higher relative expression of the $\delta$ subunit compared to females (Figure 5a, $P<$
0.0001). Relative expression of the $\alpha 5$ subunit was reduced in the preterm males compared to their term counterparts (Figure 5b, $P=0.0432$ ). Relative expression of the $\alpha 6$ subunit also appeared to be somewhat reduced in both the male and female preterm cohorts compared to their term counterparts, however, these differences in mean values did not reach significance (Figure $5 \mathrm{c}, P=0.0883$ ).

\section{Salivary Cortisol Concentration}

Salivary cortisol was assessed in juveniles before (baseline) and after (response) behavioral testing. Preterm males had higher cortisol concentrations prior to behavioral testing when compared to both term males and preterm females (Figure 6a, $P=0.0147$, and $P=0.0273$ respectively). Following behavioral testing, cortisol concentration was markedly higher in preterm females when compared to the term females (Figure $6 \mathrm{~b}$, $P=0.0483)$.

\section{DISCUSSION}

The key findings of this study were the marked behavioral differences of the preterm and term animals at an age equivalent of juvenility in the guinea pig and the associated impact 
a

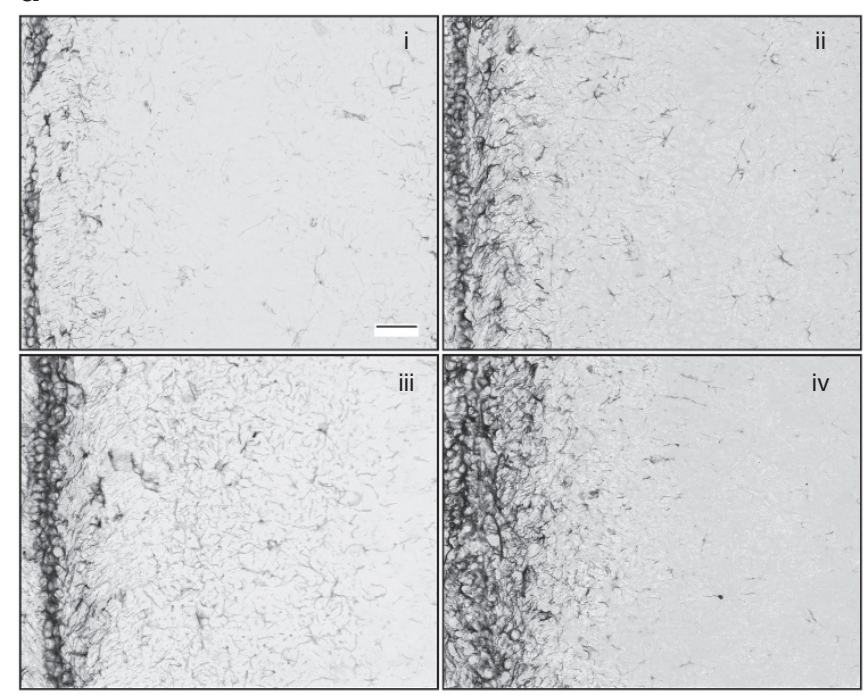

C

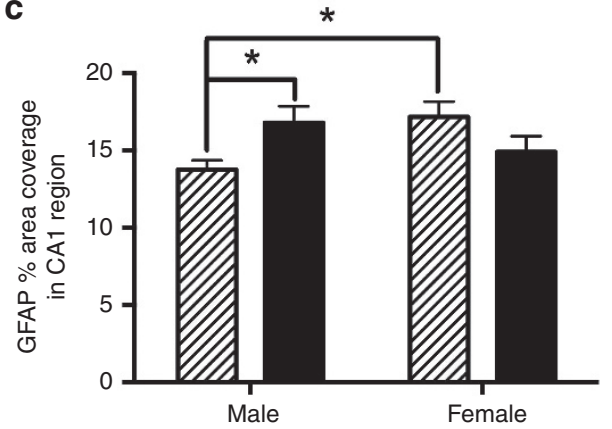

b

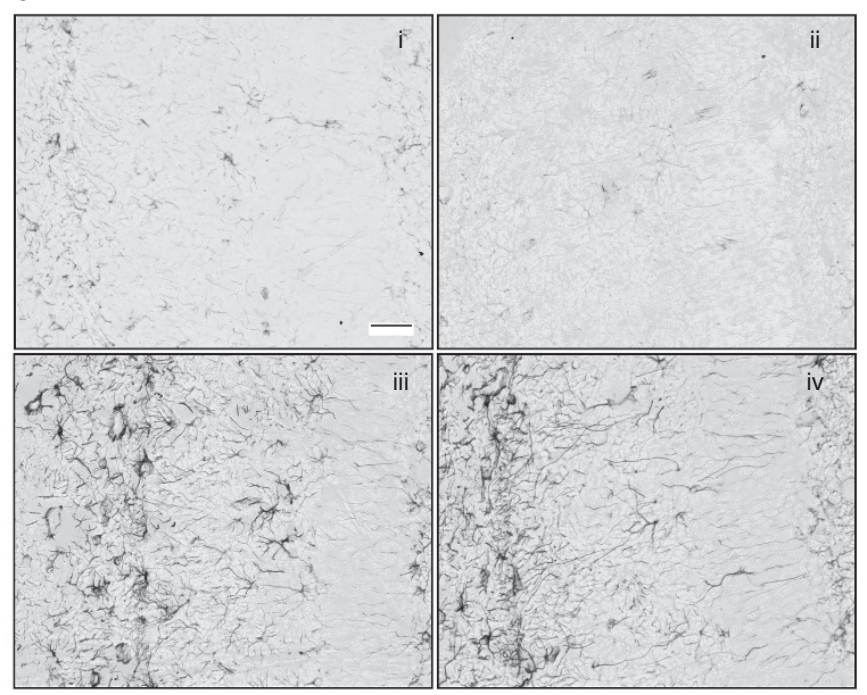

d

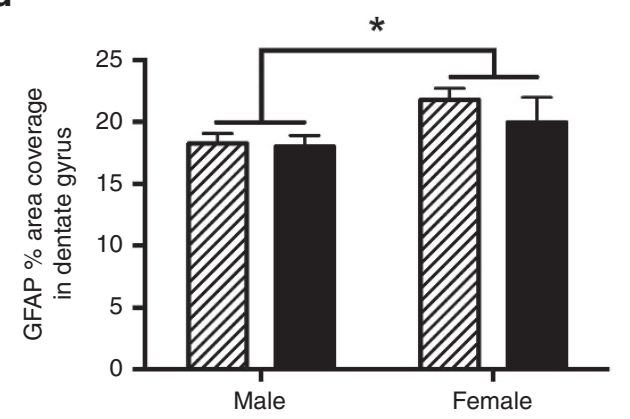

Figure 4. Glial fibrillary acidic protein immunolabeling of juvenile guinea pig brain regions. Representative photomicrographs at $20 \times$ magnification of glial fibrillary acidic protein immunolabeling and percent coverage in the juvenile guinea pig external capsule adjacent to the CA1 region of the dorsal hippocampus ( $\mathbf{a}$ and $\mathbf{c}$ ), and the dentate gyrus of the dorsal hippocampus (b and d). Scale bar = $50 \mu \mathrm{m}$; (i) male preterm, (ii) male term, (iii) female preterm, and (iv) female term for all photomicrographs. Mean \pm SEM. Preterm $=$ hashed bars, term $=$ black bars; ${ }^{*}$ indicates $P<0.05$ between bars linked by black lines. Animal groups are preterm males $=7$, term males $=8$, preterm females $=7$, and term females $=6$.

of preterm delivery on the long-term expression of $\mathrm{GABA}_{\mathrm{A}}$ receptor subunits that influence neurosteroid sensitivity. Additionally, the results indicate that there are sex differences in the influences of prematurity on these parameters, with males seemingly more affected by early delivery. Furthermore, one of the most important findings was that the deficiencies in neurodevelopment of ex-preterm males seen previously in neonates remained until juvenility, whereas females were able to restore their initial reductions $(25,26)$.

Our previous studies in guinea pigs showed markers of mature oligodendrocytes were reduced in preterm compared to term gestational age fetuses, and were markedly affected by preterm delivery in the neonatal hippocampus and subcortical white matter in both sexes $(25,26)$. The use of an ex-preterm juvenile model in the present study allowed us to ascertain whether oligodendrocyte expression remains affected until childhood, when many of the school-related disabilities become apparent clinically. The study demonstrated the lasting impact of preterm delivery on males, with reductions of oligodendrocyte immunostaining in the late developing CA1 region of the dorsal hippocampus and adjacent subcortical white matter. In this study, mature myelinating oligodendrocyte expression was reduced in the CA1 region of the hippocampus in conjunction with reactive astrocyte expression. Oligodendrocytes are the cells responsible for myelination, whereas astrocytes are seen as the support cells of the central nervous system (CNS) and one of their roles is the promotion of myelinating activity by oligodendrocytes. ATP released by astrocytes induces secretion of a regulatory protein, which then acts directly on oligodendrocytes to stimulate myelin formation (28). Astrocytes have been shown to promote myelination in vitro and also to protect the CNS from trauma by preserving myelination and reducing white matter damage in a mouse model of spinal cord injury, in addition to demonstrating myelin repair properties in a mouse knockout of astrocytes (29-31). Therefore, a reduction in myelination in the hippocampus may be a result of the reduction in reactive astrocytes, however further investigation such as determining expression of early markers of immature and preoligodendrocyte cells may aid in teasing out this relationship. Reductions in reactive astrocyte expression were also apparent in males compared to females in the dentate gyrus, which may be due to general sex-specific differences in neurodevelopment rather than an effect of preterm delivery.

Quantitation of $\mathrm{GABA}_{\mathrm{A}}$ receptor $\delta, \alpha 5$, and $\alpha 6$ subunit expression revealed that overall males exhibited increased 
a

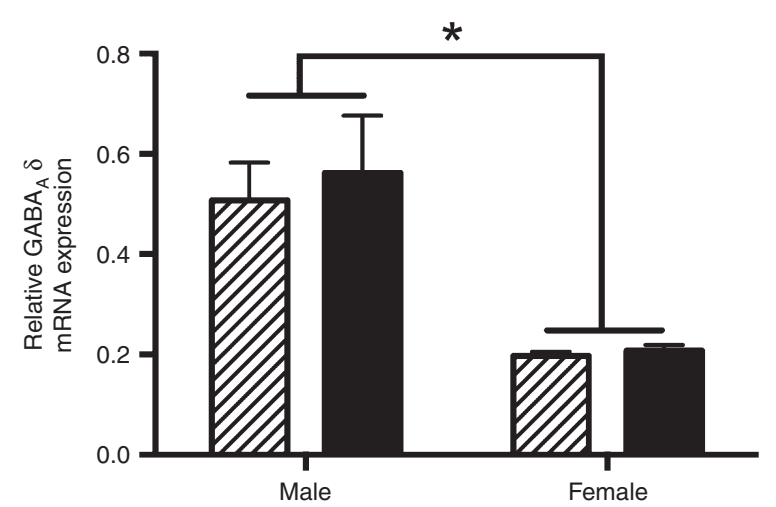

b

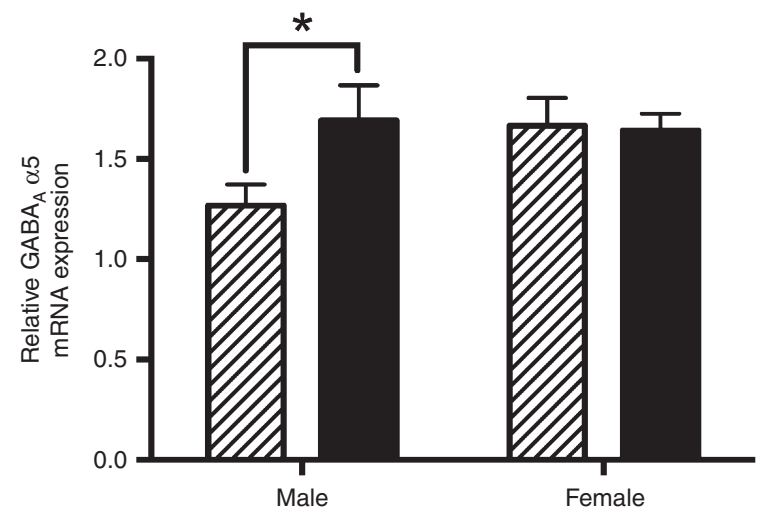

c

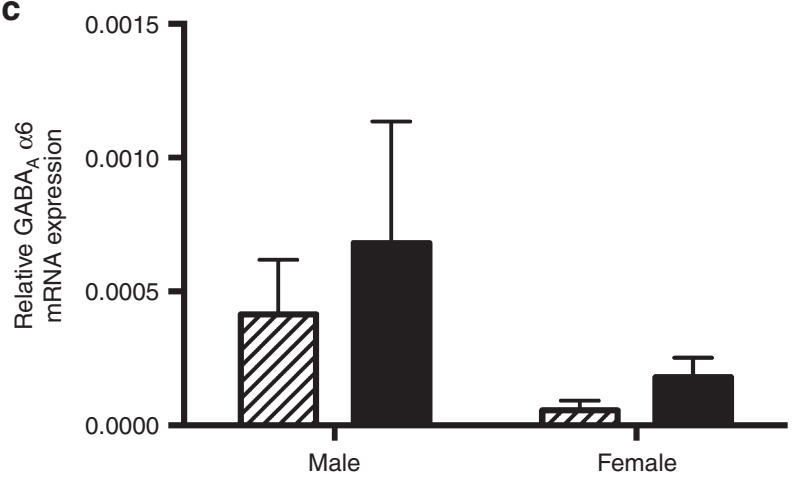

Figure 5. mRNA expression of $G A B A_{A}$ receptor subunits in juvenile guinea pig hippocampus. Relative $\mathrm{GABA}_{\mathrm{A}}$ receptor (a) $\delta$, (b) $\alpha 5$, and (c) $\alpha 6$ subunit mRNA expression in the hippocampus. Values are for preterm (62 d) and term (69 d) 28-d juvenile guinea pig hippocampal tissue. Mean \pm SEM. Preterm $=$ hashed bars, term $=$ black bars; ${ }^{*}$ indicates $P<0.05$ between bars linked by black lines. Animal groups are preterm males $=$ 10 , term males $=8$ (term males $=7$ for $\mathbf{b}$ ), preterm females $=7$, and term females $=10$ (term females $=9$ for $\mathbf{b}$ ).

expression of the $\delta$ subunit compared to females. This finding was unexpected as this subunit has a major role in allopregnanolone-receptor binding and increased levels would tend to increase allopregnanolone-mediated suppression of excitation. One possibility may be a dysregulation of neurosteroid action in response to changes in the balance of inhibitory and excitatory activity, however such possibilities require further examination. Interestingly, the expression of the $\alpha 5$ subunit was reduced in preterm males compared to the controls, with females seemingly unaffected. Animal studies have demonstrated that this subunit mediates tonic inhibition in the $\mathrm{CA} 1$ and $\mathrm{CA} 3$ of the hippocampus and is required for associative learning and memory $(32,33)$. Therefore, the reduced expression of this subunit may have implications for learning and memory in school aged ex-preterm male children. A reduction in this subunit may be affecting overall tonic inhibition and suppression of excitation in the hippocampus, and thus contributing to the hyperactivity often exhibited by expreterm males. Targeting of the $\alpha 5$ subunit is a potential avenue for treating cognitive deficits, as this has become an option for schizophrenia patients where alterations in the functionality of the GABAergic system are suggested to contribute to impaired cognition (34).

The deficiencies in the markers of neurodevelopment and neurosteroid-sensitive receptors were associated with increased activity in the open field and environment exploration tests, with the preterm males more active than the control term males. Similar results are seen in mice expressing an attention deficit hyperactivity disorder-like phenotype, where the distance travelled and time spent travelling is markedly higher for the affected mice compared to the controls (35). This hyperactive type behavior has parallels with clinical studies on expremature male children that show an increased incidence of disorders that to some extent involve hyperactivity $(7,8)$. This similarity in behavioral outcome supports the potential suitability of the guinea pig model for investigating key mechanistic links between prematurity related changes and the causation of these disorders and as a platform to investigate therapeutic treatment to target the development of these disorders. Additionally, preterm male juveniles exhibited reduced social inhibition in the familiar social testing. These findings could potentially be explained by the raised cortisol concentrations of the preterm males prior to the behavioral testing compared to the other cohorts, which suggests that they have a higher baseline level of stress than control animals. This higher cortisol baseline may be contributing to their hyperactive-type phenotype, however this relationship requires further investigation as other steroid hormones such as testosterone may be playing a role in the development of this phenotype. For all tests, the preterm females did not behave differently to the control term females, indicating that they are more resistant to effects on behavior, or are better able to adapt, despite their initial vulnerability in early life. This also parallels clinical findings that show females born preterm are more resistant to adverse outcomes compared to males, however are at higher risk of developing anxiety. Cortisol levels, and hence stress associated with the tests, suggested that the preterm females experience anxiety when introduced to the foreign behavioral testing experience as salivary cortisol was increased following the testing.

Some of the limitations that may be addressed in future studies include the preterm delivery dams receiving injections, which the term delivery dams did not, which may have introduced a degree of maternal stress due to the associated minimal pain. Prior to delivery, however, the dams were treated equally and it is unlikely this short-term minimal exposure to pain would affect the fetus shortly before delivery. Some of the 
a

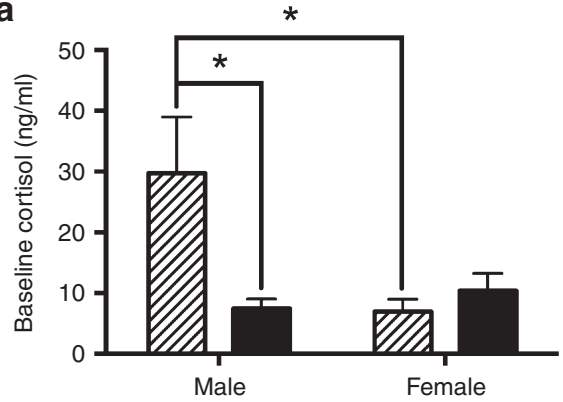

b

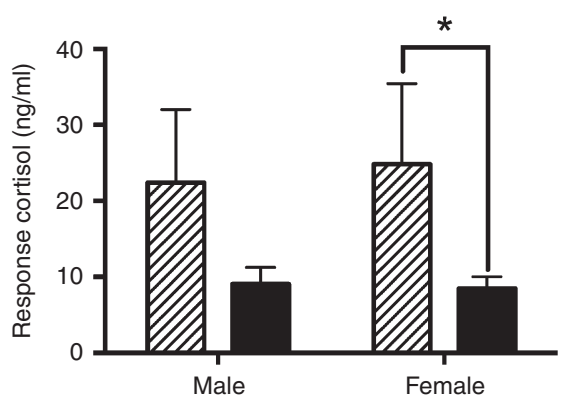

Figure 6. Salivary cortisol concentrations at baseline and in response to behavioural testing. Baseline (a) and response (b) salivary cortisol concentrations. Values are for saliva samples taken from preterm (62 d) and term (69 d) 25- $d$ juvenile guinea pigs before (baseline) and after (response) behavioral testing. Mean \pm SEM. Preterm = hashed bars, term = black bars; * indicates $P<0.05$ between bars linked by black lines. Animal numbers are preterm males $=8$ (preterm males $=9$ for $\mathbf{b}$ ), term males $=9$ (term males $=10$ for $\mathbf{b}$ ), preterm females $=6$, and term females $=10$.

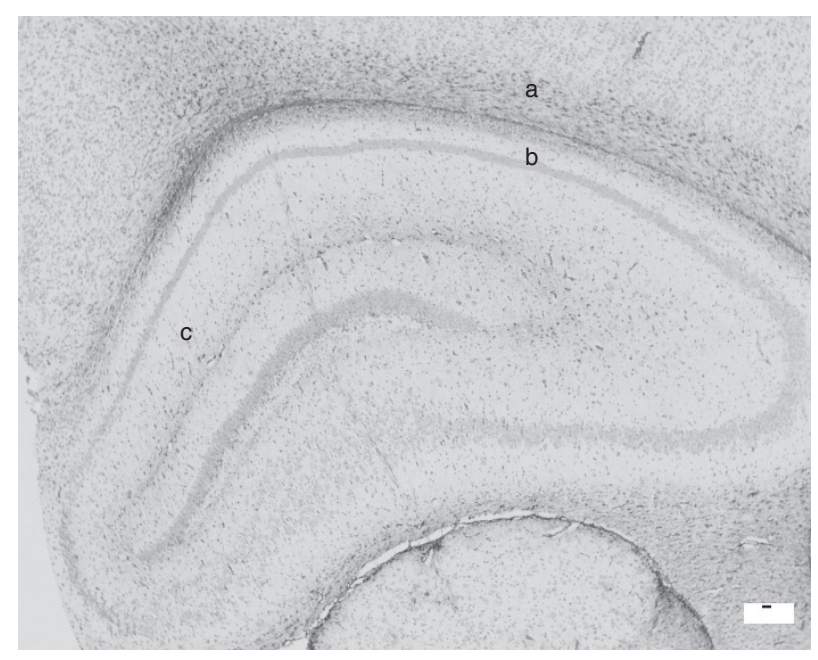

Figure 7. Representation of areas imaged for immunohistochemical analysis at $2 \times$ magnification. The above cresyl violet and glial fibrillary acidic protein-stained section highlights the specific areas of the (a) subcortical white matter, (b) CA1 region of the dorsal hippocampus, and (c) dentate gyrus.

injections that were only given to mothers delivering preterm were the two injections of betamethasone, which were given at a timing and dose that reflects current clinical practices. This minimal exposure to betamethasone is necessary for the preterm pup to survive and we suggest that any associated impact on neurosteroid synthesizing enzymes is minimal, as previous studies showing an effect have used much higher doses that were given repeatedly (36). Another essential limitation of the study was the altered treatment that the preterm pups required, including the resuscitation, nutritional supplementation, in addition to their lack of maternal grooming, which again the term delivered pups were not exposed to. These experiences are likely to have introduced an element of stress to the pups however are clinically relevant experiences for a preterm newborn to have, and thus means that our model mimics the treatment of a preterm newborn in the neonatal intensive care unit. A further unavoidable limitation was the absence of protein data for the $\mathrm{GABA}_{\mathrm{A}}$ receptor subunits examined, unfortunately due to a lack of guinea pig-specific antibodies, we were unable to obtain protein data and thus have presented the mRNA data.
Overall, this study has highlighted key late childhood differences in $\mathrm{GABA}_{\mathrm{A}}$ receptor subunit expression, myelination, and reactive astrocyte expression in the dorsal hippocampus of ex-preterm male juvenile guinea pigs, which coincided with increased salivary cortisol and a hyperactive-type phenotype. Alternatively, the females were found to be robust and seemingly unaffected by preterm delivery except for having a rise in cortisol associated with foreign situations, which parallels clinically with an increased risk of anxiety in ex-preterm females. These findings provide a basis for integral investigation of therapeutic options to protect the vulnerable ex-preterm male.

\section{METHODS}

Unless specified otherwise, all basic reagents and chemicals were supplied by Sigma Aldrich (Castle Hill, Australia).

\section{Animals}

Approval for all animal experiments and procedures carried out throughout the study was obtained from both the University of Otago and the University of Newcastle Animal Care and Ethics Committees.

Mature breeding Dunkin Hartley female guinea pigs were obtained from the University of Otago Biomedical Research Unit. Guinea pigs were housed indoors and supplied with a diet consisting of standard commercial guinea pig pellets, lucerne hay, fresh fruit, and vegetables. Pregnant dams were randomly allocated to either preterm delivery or term delivery. Dams allocated to term delivery received no further interventions during pregnancy, with pups delivered spontaneously and receiving no respiratory or nutritional support. Preterm (GA62) pups were born following preterm induction of labor as previously described (37). Briefly, dams received Betamethasone $1 \mathrm{mg} /$ kg subcutaneously (Celestone chronodose; Merck, Sharp \& Dohme, Auckland, New Zealand) 48 and $24 \mathrm{~h}$ prior to delivery to accelerate fetal lung maturation. Aglepristone $10 \mathrm{mg} / \mathrm{kg}$ subcutaneously (Provet, Auckland, New Zealand), was administered $24 \mathrm{~h}$ prior to, and on the morning of delivery to inhibit progesterone-based continuance of pregnancy. Oxytocin $3 \mathrm{IU} / \mathrm{kg}$ intramuscularly (Provet) was administered to stimulate uterine contractions, beginning $1 \mathrm{~h}$ after the second Aglepristone dose and repeated in 30-min intervals until all pups and placentas were delivered.

Resuscitation and respiratory support of pups occurred as previously described (37). Briefly, pups were placed on a heated pad and respiration encouraged. Further respiratory support was given using "Neopuff" t-piece infant resuscitator (Fisher \& Paykel, Auckland, New Zealand) with blended oxygen delivered at $5 \mathrm{l} / \mathrm{min}$. If spontaneous respiration was not achieved or sustained, positive pressure ventilation at $60 / \mathrm{min}$ with an inflation pressure of $12 \mathrm{~cm} \mathrm{H}_{2} \mathrm{O}$ and expiratory pressure of $5 \mathrm{~cm} \mathrm{H}_{2} \mathrm{O}$ was provided until spontaneous respiration was observed. Additionally, continuous positive airway pressure support at $5 \mathrm{~cm} \mathrm{H} \mathrm{H}_{2} \mathrm{O}$ was given when pups exhibited adequate respiratory 
drive but increased respiratory effort. All preterm pups were also given an initial fractional inspired oxygen concentration of $30 \%$ that was adjusted based on their colour, heart rate, and respiratory activity. Pups were housed with their mothers and littermates in a warm humidified human infant incubator (Dräger 8000 IC; Drägerwerk AG \& Co., Lübeck, Germany); ambient temperature $30^{\circ} \mathrm{C}, 70 \%$ humidity. Pups that were unable to achieve and maintain independent respiration, or showed other signs of distress (such as lack of weight gain) were excluded from the study and humanely euthanized.

Preterm pups received $0.3-0.5 \mathrm{ml}$ of replacement colostrum (Impact guinea pig colostrum replacement; Wombaroo Food Products, Adelaide, Australia) orally by a $1 \mathrm{ml}$ insulin syringe within the first hour after birth and then every $3 \mathrm{~h}$ until $24 \mathrm{~h}$ old. Between postnatal days $1-7$, pups were fed $0.5-2.0 \mathrm{ml}$ of replacement milk (Impact guinea pig milk replacement; Wombaroo Food Products) every $3 \mathrm{~h}$ or as needed to supplement independent suckling from the mother.

On postnatal day 6 ( $1 \mathrm{~d}$ prior to term equivalence), pups were transferred with their mothers from the infant incubator into a standard single cage at room temperature, and on postnatal day 7 into the nursery pen with other mothers and pups. Preterm and term neonates remained with their mothers in the nursery pen until weaning occurred at corrected PND21, after which they were placed into floor pens with animals of the same sex.

Juveniles were euthanized at corrected PND28 by sodium pentobarbitone (Lethabarb; Virbac, Milperra, Australia) $0.3 \mathrm{ml}$. At this time, body and organ weights were recorded. Each brain was sectioned down the midline in the sagittal plane to separate the two hemispheres. Each left hemisphere was fixed for immunohistochemistry, whilst the right hemisphere was further dissected and frozen in liquid nitrogen and used for further processing, including RT-PCR.

\section{Behavioral Testing}

Juveniles underwent behavioral testing at corrected PND25. A pretest saliva sample was obtained from each animal immediately prior to the first test by allowing the pup to chew a cotton bud. A post-test sample was taken no more than 30 min later following the completion of the environment exploration testing. ANY-maze tracking software version 4.7 (Stoelting, Wood Dale, Illinois) was used to analyze the videos. All salivary samples and behavioral testing was performed by staff familiar to the animals, in a designated space free from the sight, sound, or scent of other animals.

\section{Open Field and Environment Exploration Test}

The open-field test measured anxiety and locomotion and was performed as previously described (38). Guinea pig pups were allowed to explore the arena $(40 \times 40 \mathrm{~cm})$ for $10 \mathrm{~min}$, with entries into the inner zone (an area $20 \times 20 \mathrm{~cm}$ in the centre of the arena) recorded.

The environment exploration test measured the animals' exploratory behavior and anxiety (38). Following the open-field test, the animal was removed from the arena and two identical objects were placed in the center of the top two quadrants of the arena. The animal was then placed back into the arena and allowed to investigate the objects for $10 \mathrm{~min}$.

Parameters measured included distance travelled in the entire arena and total time mobile, in addition to distance travelled and time spent mobile within the inner zone, and time spent investigating the foreign objects.

\section{Familiar Social Test}

The familiar social test measured social interactions towards a familiar animal. The test animal was placed into the arena with an animal of the same sex and age from the same home pen. For $5 \mathrm{~min}$, the pair of animals were allowed to interact. Parameters measured included approaching, and having an affectionate or agonistic interaction with the familiar animal.

\section{Immunohistochemistry}

Myelination and reactive astrocyte expression was examined in the CA1 region of the dorsal hippocampus, in addition to the adjacent subcortical white matter and dentate gyrus (Figure 7). Immunohistochemistry was performed on 8- $\mu \mathrm{m}$ sections of paraffin-embedded brains that were cut using a Leica RM2145 Microtome (Leica Microsystems Pty, North Ryde, Australia) $(25,39)$. Tissues were dewaxed, incubated in citrate buffer ( $\mathrm{pH}$ 6.0) and phosphate-buffered saline containing 3\% hydrogen peroxide, and blocked for $1 \mathrm{~h}$ at room temperature in Bovine serum albumin blocking solution ( $0.5 \% \mathrm{w} / \mathrm{v}$ bovine serum albumin, $0.05 \% \mathrm{w} / \mathrm{v}$ Saponin, $0.05 \% \mathrm{v} / \mathrm{v}$ Sodium Azide in $0.1 \mathrm{M}$ phosphate-buffered saline). Incubation in primary antibodies (MBP, M9434; and GFAP, G3893) and secondary antibodies (biotinylated anti-rat IgG, B7139; and antimouse IgG, B6649 respectively) were performed before tertiary incubation in streptavidin-biotin-horseradish peroxidase complex. To reveal the immunolabeling, incubation in 3,3'-diaminobenzidine tetrahydrochloride solution (Metal Enhanced DAB Substrate Kit; ThermoFisher Scientific, Scoresby, Australia) occurred.

Stained slides were imaged using the Aperio imaging system (Leica Biosystems). Percent area coverage at $20 \times$ magnification was used to quantify staining. ImageJ version 1.47 (National Institutes of Health, Bethesda, MD) was used to calculate area coverage by converting to gray-scale and then binary, and manually adjusting threshold based on the original stained image $(25,39)$. An overall average of area coverage staining was calculated by taking the average of three images captured from two consecutive sections per animal. Sections of only the highest standard were used for analysis, with poorer sections excluded from the study.

\section{Real-Time PCR}

RT-PCR was performed as previously described $(25,36)$. Frozen hippocampal tissue was homogenized in RLT Plus Buffer (Qiagen RNeasy Plus Mini Kit; Qiagen Pty, Chadstone Centre, Australia) using a Precellys 24 dual tissue homogenizer (Bertin Technologies, Provence, France). RNA was extracted using the Qiagen RNeasy Plus Mini Kit. Samples with poor RNA A260/280 ratios and integrity on an RNA gel were not used for further analysis.

Superscript III Reverse Transcription kit (Invitrogen, Carlsbad, CA) was used to synthesize cDNA on the GeneAmp 9700 PCR machine (Applied Biosystems, Life Technologies Pty, Mulgrave, Australia). RT-PCR was performed using a 7500 ABI real-time machine (Applied Biosystems), for primer pairs $(\delta, \alpha 5$, and $\alpha 6)$ and the housekeeping gene $\beta$-actin (25). Each sample was run in duplicate along with an associated negative control sample. Products were detected using the SYBR Green (Applied Biosystems) DNA binding dye method. Results were analyzed by Sequence Detection Software v2.01 (Applied Biosystems) and the comparative Ct method $\left(2^{-\Delta \Delta \mathrm{Ct}}\right)$ was used to calculate relative fold change. Controls included $\beta$-actin and a calibrator, which consisted of pooled brain samples and was used across all plates. Consistent Ct values were obtained for $\beta$-actin across the term/preterm and male/female samples.

\section{Cortisol Enzyme-Linked Immunosorbent Assay}

The Salimetrics Salivary Cortisol Assay (Salimetrics, State College, $\mathrm{PA}$ ) was used to measure cortisol concentrations in guinea pig saliva samples. As previously described, the sensitivity of the assay is $0.012-$ $3.0 \mu \mathrm{g} / \mathrm{dl}$, with the inter- and intra-assay coefficients of variance 6.89 and $5.52 \%$ respectively $(40)$.

\section{Statistical Analysis}

Data was analyzed by two-way ANOVA and unpaired $t$-tests in samesex groups using Graphpad Prism software (version 6.01; Graphpad Software, La Jolla, CA). When a significant difference was found, Tukey post-hoc tests and corrections for multiple comparisons were performed. Unless otherwise stated, all data are expressed as mean \pm SEM and significance considered as $P<0.05$.

\section{ETHICAL STANDARDS}

The authors assert that all animal work performed complies with the ethical standards of the relevant national guides on the care and use of laboratory animals (National Animal Ethics Advisory Committee of New Zealand, and the National Health and Medical Research Council Australian Code of Practice for the Care and Use of Animals for Scientific Purposes), and has been approved by the institutional committees (University of Otago, Wellington Animal Ethics Committee, and the University of Newcastle Animal Care and Ethics Committee).

\section{ACKNOWLEDGMENTS}

We would like to acknowledge Katherine Wright and Mike Peebles for their contributions to the animal work. 


\section{STATEMENT OF FINANCIAL SUPPORT}

This study was funded by the National Health and Medical Research Council (NHMRC) (grant number APP1003517) (Newcastle, Australia) and by funds from the Department of Paediatrics and Child Health, University of Otago (Wellington, New Zealand), and project grants awarded to M.J.B. from the University of Otago, The Neonatal Trust, and The Royal Australasian College of Physicians (Wellington, New Zealand).

Disclosure: The authors confirm that there are no financial ties to products, or conflicts of interest to disclose.

\section{REFERENCES}

1. Mathews TJ, Menacker F, MacDorman MF. Infant mortality statistics from the 2000 period linked birth/infant death data set. Natl Vital Stat Rep 2002;50:1-28.

2. Moster D, Lie RT, Markestad T. Long-term medical and social consequences of preterm birth. N Engl J Med 2008;359:262-73.

3. Huddy CL, Johnson A, Hope PL. Educational and behavioural problems in babies of 32-35 weeks gestation. Arch Dis Child Fetal Neonatal Ed 2001;85:F23-8.

4. Kirkegaard I, Obel C, Hedegaard M, Henriksen TB. Gestational age and birth weight in relation to school performance of 10 -year-old children: a follow-up study of children born after 32 completed weeks. Pediatrics 2006;118:1600-6.

5. Chyi LJ, Lee HC, Hintz SR, Gould JB, Sutcliffe TL. School outcomes of late preterm infants: special needs and challenges for infants born at 32 to 36 weeks gestation. J Pediatr 2008;153:25-31.

6. Cheong JL, Doyle LW. Increasing rates of prematurity and epidemiology of late preterm birth. J Paediatr Child Health 2012;48:784-8.

7. Lindström K, Lindblad F, Hjern A. Preterm birth and attention-deficit/ hyperactivity disorder in schoolchildren. Pediatrics 2011;127:858-65.

8. Linnet KM, Wisborg K, Agerbo E, Secher NJ, Thomsen PH, Henriksen TB. Gestational age, birth weight, and the risk of hyperkinetic disorder. Arch Dis Child 2006;91:655-60.

9. Singh GK, Kenney MK, Ghandour RM, Kogan MD, Lu MC. Mental health outcomes in US children and adolescents born prematurely or with low birthweight. Depress Res Treat 2013;2013:570743.

10. Gurka MJ, LoCasale-Crouch J, Blackman JA. Long-term cognition, achievement, socioemotional, and behavioral development of healthy latepreterm infants. Arch Pediatr Adolesc Med 2010;164:525-32.

11. van Baar AL, Vermaas J, Knots E, de Kleine MJ, Soons P. Functioning at school age of moderately preterm children born at 32 to 36 weeks' gestational age. Pediatrics 2009;124:251-7.

12. Volpe JJ. Brain injury in premature infants: a complex amalgam of destructive and developmental disturbances. Lancet Neurol 2009;8:110-24.

13. Rees S, Inder T. Fetal and neonatal origins of altered brain development. Early Hum Dev 2005;81:753-61.

14. Counsell SJ, Rutherford MA, Cowan FM, Edwards AD. Magnetic resonance imaging of preterm brain injury. Arch Dis Child Fetal Neonatal Ed 2003;88:F269-74.

15. Hirst JJ, Palliser HK, Yates DM, Yawno T, Walker DW. Neurosteroids in the fetus and neonate: potential protective role in compromised pregnancies. Neurochem Int 2008;52:602-10.

16. Crossley KJ, Walker DW, Beart PM, Hirst JJ. Characterisation of GABA(A) receptors in fetal, neonatal and adult ovine brain: region and age related changes and the effects of allopregnanolone. Neuropharmacology 2000;39:1514-22.

17. Crossley KJ, Nitsos I, Walker DW, Lawrence AJ, Beart PM, Hirst JJ. Steroidsensitive GABAA receptors in the fetal sheep brain. Neuropharmacology 2003;45:461-72.

18. Belelli D, Harrison NL, Maguire J, Macdonald RL, Walker MC, Cope DW. Extrasynaptic GABAA receptors: form, pharmacology, and function. J Neurosci 2009;29:12757-63.

19. Mihalek RM, Banerjee PK, Korpi ER, et al. Attenuated sensitivity to neuroactive steroids in gamma-aminobutyrate type A receptor delta subunit knockout mice. Proc Natl Acad Sci USA 1999;96:12905-10.
20. Spigelman I, Li Z, Liang J, et al. Reduced inhibition and sensitivity to neurosteroids in hippocampus of mice lacking the $\mathrm{GABA}(\mathrm{A})$ receptor delta subunit. J Neurophysiol 2003;90:903-10.

21. Sanacora G, Gueorguieva R, Epperson CN, et al. Subtype-specific alterations of gamma-aminobutyric acid and glutamate in patients with major depression. Arch Gen Psychiatry 2004;61:705-13.

22. Merali Z, Du L, Hrdina P, et al. Dysregulation in the suicide brain: mRNA expression of corticotropin-releasing hormone receptors and GABA(A) receptor subunits in frontal cortical brain region. J Neurosci 2004;24:1478-85.

23. Hasler G, Nugent AC, Carlson PJ, Carson RE, Geraci M, Drevets WC. Altered cerebral gamma-aminobutyric acid type A-benzodiazepine receptor binding in panic disorder determined by [11C]flumazenil positron emission tomography. Arch Gen Psychiatry 2008;65:1166-75.

24. Nutt DJ, Malizia AL. Structural and functional brain changes in posttraumatic stress disorder. J Clin Psychiatry 2004;65 Suppl 1:11-7.

25. Shaw JC, Palliser HK, Walker DW, Hirst JJ. Preterm birth affects GABAA receptor subunit mRNA levels during the foetal-to-neonatal transition in guinea pigs. J Dev Orig Health Dis 2015;6:250-60.

26. Kelleher MA, Hirst JJ, Palliser HK. Changes in neuroactive steroid concentrations after preterm delivery in the Guinea pig. Reprod Sci 2013;20: 1365-75.

27. Palliser HK, Kelleher MA, Tolcos M, Walker DW, Hirst JJ. Effect of postnatal progesterone therapy following preterm birth on neurosteroid concentrations and cerebellar myelination in guinea pigs. J Dev Orig Health Dis 2015;6:350-61.

28. Ishibashi T, Dakin KA, Stevens B, et al. Astrocytes promote myelination in response to electrical impulses. Neuron 2006;49:823-32.

29. Faulkner JR, Herrmann JE, Woo MJ, Tansey KE, Doan NB, Sofroniew MV. Reactive astrocytes protect tissue and preserve function after spinal cord injury. J Neurosci 2004;24:2143-55.

30. Moore CS, Milner R, Nishiyama A, et al. Astrocytic tissue inhibitor of metalloproteinase-1 (TIMP-1) promotes oligodendrocyte differentiation and enhances CNS myelination. J Neurosci 2011;31:6247-54.

31. Sorensen A, Moffat K, Thomson C, Barnett SC. Astrocytes, but not olfactory ensheathing cells or Schwann cells, promote myelination of CNS axons in vitro. Glia 2008;56:750-63.

32. Crestani F, Keist R, Fritschy JM, et al. Trace fear conditioning involves hippocampal alpha5 GABA(A) receptors. Proc Natl Acad Sci USA 2002;99:8980-5.

33. Glykys J, Mody I. Hippocampal network hyperactivity after selective reduction of tonic inhibition in GABA A receptor alpha5 subunit-deficient mice. J Neurophysiol 2006;95:2796-807.

34. Gill KM, Grace AA. The role of $\alpha 5$ GABAA receptor agonists in the treatment of cognitive deficits in schizophrenia. Curr Pharm Des 2014;20: 5069-76.

35. Kim P, Choi CS, Park JH, et al. Chronic exposure to ethanol of male mice before mating produces attention deficit hyperactivity disorder-like phenotype along with epigenetic dysregulation of dopamine transporter expression in mouse offspring. J Neurosci Res 2014;92:658-70.

36. McKendry AA, Palliser HK, Yates DM, Walker DW, Hirst JJ. The effect of betamethasone treatment on neuroactive steroid synthesis in a foetal Guinea pig model of growth restriction. J Neuroendocrinol 2010;22: $166-74$.

37. Berry M, Gray C, Wright K, Dyson R, Wright I. Premature guinea pigs: a new paradigm to investigate the late-effects of preterm birth. J Dev Orig Health Dis 2015;6:143-8.

38. Bennett GA, Palliser HK, Shaw JC, Walker D, Hirst JJ. Prenatal stress alters hippocampal neuroglia and increases anxiety in childhood. Dev Neurosci 2015;37:533-45.

39. Kelleher MA, Palliser HK, Walker DW, Hirst JJ. Sex-dependent effect of a low neurosteroid environment and intrauterine growth restriction on foetal guinea pig brain development. J Endocrinol 2011;208:301-9.

40. Bennett GA, Palliser HK, Saxby B, Walker DW, Hirst JJ. Effects of prenatal stress on fetal neurodevelopment and responses to maternal neurosteroid treatment in Guinea pigs. Dev Neurosci 2013;35:416-26. 